\title{
HISTORIA Y COMBINATORIA \\ DE LAS REPRESENTACIONES CIENTÍFICAS. COMENTARIOS A LA PROPUESTA DE IBARRA Y MORMANN
}

\author{
Sergio F. Martínez \\ Instituto de Investigaciones Filosóficas \\ UNAM \\ sfmar@servidor.unam.mx
}

RESUMEN: En este texto se examina críticamente la teoría combinatoria de las representaciones científicas de Andoni Ibarra y Thomas Mormann. El núcleo de la crítica va dirigido a mostrar que una serie de estudios sobre la ciencia, que ellos mismos mencionan, sugiere que la clasificación en tipos de representaciones propuesta es problemática. Es más, esos mismos estudios muestran que por lo menos muchas representaciones tienen una dimensión histórica que parece imposible capturar por medio del tipo de formalismo propuesto.

PALABRAS CLAVE: representación, enfoque formalista y enfoque historicista

SUMMARY: Ibarra and Mormann's combinatorial theory of scientific representations is subject to critical scrutiny. It is argued that the classification of types of representations offered cannot serve as a basis for an analysis of the variety of representations that happen to arise in science. Empirical studies on ways of representing used in science strongly suggest that a formal theory of representations of the kind proposed is not able to capture the historical dimension of the structure of representations.

KEY WORDS: representation, formalist account and historicist account

El tema de la representación ha tenido importantes vaivenes a lo largo de la historia de la filosofía: en la tradición cartesiana ha sido uno de los asuntos centrales de la filosofía; en la tradición pragmatista y en la tradición analítica, sobre todo de corte davidsoniano, ha pasado a ser un tema subordinado al de la interpretación. Por representación se entienden muchas cosas, en parte porque el tema está íntimamente ligado a otras cuestiones resbalosas; tiene que ver, por ejemplo, con diferentes maneras de entender lo que es un concepto, y con teorías de 
la referencia. El trabajo de Ibarra y Mormann ${ }^{1}$ que quiero comentar en estas páginas busca contribuir a la revalorización del concepto de representación en la ciencia. Los autores consideran que la representación es un concepto complejo y "difícil" que requiere elaboración y elucidación, lo que, según ellos, equivale a decir que "es un concepto que para su comprensión necesita una teoría de las representaciones científicas" (p. 4). El núcleo del trabajo consiste en la elaboración de un concepto general de representación que, según los autores, capta las propiedades esenciales de las representaciones científicas.

La propuesta de Ibarra y Mormann parte de una clasificación de lo que consideran que son las principales formas de la representación "identificables en las prácticas representacionales de la ciencia":

1) La representación como isomorfía

2) La representación como sustitución

3) La representación como homomorfía

4) La representación como homología

El primer tipo de representación se caracteriza por la necesidad de que exista una semejanza entre los dos relatos de la representación. Se dice que este enfoque se menciona sólo por mor de la completud, porque de hecho no desempeña ningún papel relevante en la práctica científica (p. 9). La representación como sustitución se caracteriza en términos de ejemplos. Se da el ejemplo del embajador de un país que puede representarlo en una conferencia; un abogado que representa a su cliente; el papel del apoderado de un menor. Se da también el ejemplo de los números y otras magnitudes matemáticas "que funcionan como sustitutos vicariales de entidades empíricas de diverso tipo" (p. 9). La representación como homomorfía corresponde a la concepción de la representación "como aplicación preservadora

1 Véase A. Ibarra y T. Mormann, "Una teoría combinatoria de las representaciones científicas", Crítica, vol. XXXII, no. 95, 2000, pp. 3-46. En adelante, las páginas que aparecen entre paréntesis proceden de este artículo. 
de estructura" (p. 10). La representación como homología se caracteriza primero negativamente, como aquel tipo de representación que no depende ni de la semejanza objetual ni de la estructural. Constructivamente la idea se introduce a partir de una idea de Hertz (a la que me referiré más adelante).

Los autores pretenden mostrar que las representaciones científicas no son generalmente de tipo homomórfico, es decir, del tipo de representación que, según ellos, ha sido el más estudiado en la filosofía de la ciencia, sino de tipo homológico. Una parte importante del proyecto consiste en caracterizar ese último tipo de representación, y en hacer ver su capacidad para sistematizar el papel de la representación en la ciencia. Según los autores, la idea de la representación como homología puede derivarse de la descripción que hace Hertz de la actividad científica como la producción de una simetría entre "las consecuencias naturalmente necesarias" y "las consecuencias intelectualmente necesarias" de sus representaciones. Esta simetría se ilustra en el siguiente diagrama:

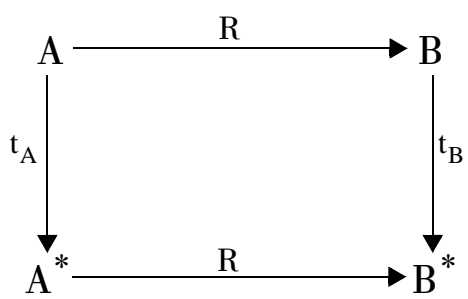

La idea de los autores es la siguiente:

la parte izquierda del diagrama, $A \stackrel{t_{\mathrm{A}}}{\longrightarrow} A^{*}$ puede interpretarse como un proceso empírico en el que se pasa del estado $A$ al estado $A^{*}$ a través de $t_{\mathrm{A}}$. Este paso se representaría por un proceso teórico consistente en el paso de $B$ a $B^{*}$ por $t_{\mathrm{B}}$, tal que $t_{\mathrm{B}}$ es una relación lógica $[\ldots]$ entre $B$ y $B^{*}$ que corresponde a la re- 
lación $t_{\mathrm{A}}$ "naturalmente necesaria" en el sentido que el diagrama conmuta. (p. 14)

Otro aspecto importante de su propuesta es que todas las representaciones en la ciencia forman una compleja red representacional que está constituida por diversos tipos de combinaciones. Un objeto o proceso, por ejemplo, puede generar diferentes representaciones o una representación puede valer para diferentes objetos, procesos o representaciones. Esta estructura reticular de las representaciones se podría capturar por medio de la estructura combinatoria (asociativa) de los diagramas. La caracterización formal de este aspecto combinatorio se logra al afirmar que tanto para las representaciones preservadoras de estructura como para las representaciones homólogas se cumple que las representaciones $f: A \rightarrow B, g: B \rightarrow C$ pueden combinarse como $h: A \rightarrow C$. Los autores consideran que puede admitirse sin problemas que esta combinación o concatenación es asociativa; esto es, que satisface

$$
f \cdot(g \cdot h)=(f \cdot g) \cdot h
$$

Usando estos supuestos no les es difícil mostrar que la teoría matemática de las categorías es de hecho una teoría de la combinación de representaciones posibles. Una vez que este punto ha sido establecido, los autores pasan a mostrar la significación epistemológica de la teoría de categorías concebida como una teoría combinatoria general de las representaciones.

Es indudable la importancia que tiene este tipo de investigaciones. El concepto de representación es un concepto que requiere revalorización en la filosofía de la ciencia. El énfasis que los autores ponen en el carácter interdependiente de las representaciones, y en su carácter reticular, me parece muy atinado y sugerente. Este carácter reticular de las representaciones es uno de los temas más explotados en una gran cantidad de estudios empíricos sobre la naturaleza de la representación científica, y muy poco se ha dicho sobre las implicaciones de esos estudios para la filosofía. El artículo que estoy comentando es uno de los pocos estudios en la literatura reciente que tratan de insertar esa literatura en un proyecto filosófico, y este hecho es suficiente 
para tomar muy en serio lo que los autores dicen. Sin embargo, me parece que el proyecto, en su presentación actual, adolece de problemas serios a los que me refiero a continuación. Cabe aclarar que Ibarra y Mormann reconocen que están presentando un proyecto en una etapa inicial, y que, por lo tanto, se debe entender que los comentarios siguientes se encaminan más bien a sugerir temas sobre los que tendría que decirse algo, que a una refutación del proyecto.

Antes de entrar a lo que va a ser el tema central de mis comentarios quiero mencionar dos problemas con el tipo de formalización propuesta. En primer lugar, con respecto al tema de la asociatividad de las representaciones. La función de exponenciación de números reales es sumamente importante en la ciencia contemporánea. En muchas disciplinas existen ejemplos de sistemas físicos que se modelan por medio de procesos exponenciales, los cuales incluyen problemas de crecimiento de poblaciones en biología, circuitos eléctricos, decaimiento de sistemas radiactivos y absorción de ondas en medios lineales en física. Las combinaciones de representaciones de sistemas físicos modelados por un sistema exponencial no satisfacen la condición de asociatividad. Podría argumentarse que este tipo de combinaciones no tienen significado físico; pero no parece haber nada que impida que pueda hablarse del crecimiento exponencial en un capacitor que actúa como fuente de otro capacitor, o de un modelo del crecimiento exponencial de una población que a su vez depende de alimentación con decrecimiento exponencial. En todo caso, no puede decidirse a priori si esto es o no posible, en aras de una teoría general de la representación.

Otro punto relacionado con el formalismo utilizado es el siguiente: los autores consideran que la teoría de categorías nos permite hablar de representaciones que no preservan estructuras, sino relaciones lógicas; pero esta manera de ponerlo sugiere algo incorrecto. Es perfectamente posible definir las relaciones lógicas como estructura (como parte de la estructura de un topo) y, por lo tanto, utilizar la preservación de estructura para preservar relaciones lógicas. Esto sugiere que sería necesario justificar, bastante más de lo que se hace en este trabajo, la tesis de que las representaciones como homología son más generales que 
las representaciones homomórficas (por lo menos en el sentido de que las representaciones homológicas no preservan objetos ni estructuras, sino relaciones lógicas; véase el recuadro en la p. 18).

Luego de estos breves comentarios sobre el uso del formalismo de la teoría de categorías paso al tema central de mi comentario. Quiero mostrar que si bien Ibarra y Mormann sugieren que los trabajos hechos en estudios sobre la ciencia en relación con el tema de la representación apuntan en la dirección de su propuesta, muy por el contrario, considero que estos trabajos muestran que este tipo de enfoque formalista del tema de la representación tiene serias limitaciones.

Comenzaré con un poco de historia. Un importante supuesto de la filosofía desde el siglo XVII hasta el siglo XIX es la doctrina de que en la representación uno debería identificar tantas partes como las hay en el objeto representado (que, por supuesto, debería contar como una manera de formular el principio básico de la tradición de representación isomorfista). Esta doctrina, expresada a través de las diferentes versiones de la teoría asociacionista de las ideas, llega hasta Kant. Pero ya para Kant queda muy claro que una representación no es una simple copia de la realidad; Kant señala que uno puede decir que las notas de una pieza musical son una representación de la conexión armónica de los tonos, no porque cada nota sea similar a cada tono, sino porque las notas están conectadas una a la otra precisamente como lo están los tonos. Lo que sí es cierto es que en Kant no se hace todavía de manera explícita una distinción que va a desempeñar un papel muy importante en la filosofía de la ciencia del siglo Xx, y que parece desempeñar también un papel importante en la propuesta de Ibarra y Mormann: la distinción entre representaciones objetivas y subjetivas.

A partir de Bolzano se distingue entre representaciones subjetivas y representaciones objetivas. Representaciones subjetivas son aquellas que los psicologistas consideran como estados mentales o "determinaciones del alma", por ejemplo, cuando percibo un objeto físico. Las representaciones objetivas constituyen el contenido de las representaciones subjetivas. Desde el positivismo lógico, la filosofía de la ciencia ha utilizado esta distinción y 
ha asumido que a la filosofía de la ciencia sólo le interesan las representaciones objetivas; la idea de una epistemología objetiva de Popper, la epistemología de Carnap basada en la distinción entre preguntas internas y externas, e incluso los conceptos madeja de Neurath ("Ballungen"), muestran la importancia que se le otorga a las representaciones objetivas en la filosofía de la ciencia del siglo Xx (aunque como mencionaré más adelante, dentro de la tradición historicista siempre hay ambigüedades interesantes). La importancia de este carácter objetivo y público de las representaciones científicas se recalca en la filosofía analítica a través del cambio de terminología que Hertz introduce entre "Vorstellung" y "Darstellung". "Darstellung" es representación en el sentido en que hablamos de "representación teatral", o en el sentido en que un avión para armar es un modelo (a escala) de cierto tipo de avión. Representar un fenómeno en este sentido es mostrarlo a un público, exhibirlo, presentarlo en determinado contexto que se considera públicamente evaluable.

En la filosofía de la ciencia de corte historicista surgen naturalmente cuestionamientos de peso a la importancia metodológica que tiene esta distinción entre representaciones objetivas y subjetivas. En los estudios sobre la ciencia, este cuestionamiento se torna en abierta rebeldía. Aquí ya claramente se niega que haya una distinción epistemológica o metodológicamente útil entre representaciones objetivas y subjetivas. Latour es muy claro al respecto: "out there", "nature"; "in there", "the mind", “... all pertain to the same settlement, a settlement that can be replaced by several alternatives" (Latour 1999, p. 14). En otras palabras, lo que se entienda por representaciones objetivas va a depender de un contexto histórico, es el producto contingente de una determinada historia, e involucra de manera importante elementos representacionales que usualmente se consideran subjetivos.

Esta breve reseña de la historia del concepto de representación ya es suficiente para sugerir maneras en las que la clasificación de las formas de representación en la ciencia que presentan Ibarra y Mormann deja mucho que desear. Es indudable que el problema es importante, y que propuestas de clasificaciones no hay muchas; por lo general, sólo se identifica una noción alternativa contra la cual se establece una oposición, y a partir de 
esa oposición se defiende cierta idea de representación. Pero la clasificación que los autores ofrecen me parece confusa en varios aspectos. En primer lugar, no queda claro qué tipo de clasificación están ofreciendo; según ellos, se trata de una clasificación motivada por razones esencialmente metodológicas, pero nunca queda claro cuáles son esas razones, más bien parece que las motivaciones son metafísicas.

Como ejemplo de representación isomórfica se ofrece el mecanismo descrito por la teoría fisiológica de la percepción cartesiana. Si éste es un ejemplo paradigmático de lo que se entiende por representación isomórfica, entonces es evidente que ya no desempeña ningún papel en la práctica científica. Ciertamente, si la idea es que la representación requiere una relación entre lo similar (que es a lo que parecen referirse los autores cuando hablan de "imagen especular"), entonces la idea puede rechazarse con muy buena base, como bien lo ha dicho Nelson Goodman en una crítica famosa: "Similarity is insiduos [...] is a pretender, an impostor, a quack" (Goodman 1972, p. 437). Pero es muy importante no confundir similitud con semejanza. Y no veo por qué no hablar de isomorfismo en relación con semejanza. Después de todo, en matemáticas, un isomorfismo es un caso especial de homomorfismo.

Es más, para referirme sólo a dos autores ya mencionados, Latour y Galison, ambos hablan de prácticas representacionales que prima facie por lo menos deben clasificarse en la tradición isomórfica. La tradición de la imagen en la física del siglo XX de la que habla Galison, por ejemplo, se caracteriza en términos de la relación entre un estado interno sólo si una situación externa particular tiene lugar (Galison 1997, p. 25). Esto debería contar como un ejemplo de la tradición isomorfista, si acaso hay alguno; pero entonces sería obviamente falso lo que se afirma en el texto: que la representación como isomorfismo "no desempeña ningún papel relevante en la práctica científica" (p. 9). Los autores podrían argüir que esta tradición de representación no concuerda con la caracterización que se hace de la representación por isomorfía en la misma página 9, donde dice que se requiere "la necesaria existencia de una semejanza entre los dos relatos de la representación". Pero si éste es el requisito, ni el ejemplo 
de los tonos musicales de Kant que mencionamos anteriormente lo satisfaría, y entonces se estaría caracterizando como tradición isomórfica un espantapájaros. Por otro lado, tanto el ejemplo de Kant como la tradición de la imagen estarían de acuerdo con la "identificabilidad de semejanzas" de la que se habla en el siguiente párrafo del texto de Ibarra y Mormann (p. 9) como distintivo de la tradición isomórfica.

Ahora bien, hay otro asunto de más fondo: los autores parecen tener la idea de estar presentando una clasificación subespecie aeternitates, idea que los lleva a decir que si bien ya no está en uso, la representación isomórfica es un tipo de representación científica que tiene que mencionarse porque tiene un lugar en el olimpo de los tipos. Si no se supone algo así, sería obvio que tendría que decirse mucho más para caracterizar el abanico de maneras de representar que pueden caber dentro de la caracterización de "representación isomórfica" a lo largo de la historia de la ciencia y la filosofía.

Por otro lado, los autores parecen estar generando una falsa oposición entre la tradición homológica y otras tradiciones de representación. Para precisar ideas tomemos como ejemplo la filosofía de la ciencia de Toulmin (Toulmin 1972), basada en la idea de la existencia de una gran variedad de "procedimientos explicativos", en la caracterización de los cuales el concepto de técnicas de representación cumple un papel importante. Toulmin es muy claro: en su teoría, "representación" debe entenderse en el sentido de "Darstellung" y no en el sentido de "Vorstellung" (i.e. en el sentido subjetivo de representación):

and if we here pick on "techniques of representation" as a key element in the meaning (or collective use) of scientific concepts, we may emphasize that the term "representation" is to be understood solely in the "Darstellung" sense [...] (Hertz , p. 196)

Para Toulmin, sin embargo, las técnicas de representación pueden ser tan variadas como las herramientas humanas. Así como no es razonable pensar que es posible encontrar una "teoría general de las herramientas", tampoco lo es pretender encontrar "una teoría general de las representaciones". 
El pensamiento de Toulmin, además, es un buen ejemplo de cómo el concepto de representación objetiva de Hertz, representación en el sentido de "Darstellung", no en el sentido de "Vorstellung", no tiene necesariamente que asociarse con la idea de que las representaciones son homológicas. Toulmin también estaría muy de acuerdo en que las representaciones vienen en combinaciones complejas; lo que Toulmin llama transmits, por ejemplo, son ese tipo de combinación. Las representaciones de Toulmin tendrían o podrían tener elementos de las diferentes formas que Ibarra y Mormann proponen, y en particular serían representaciones vicariantes (o sustitutivas) con elementos homomórficos. Como ya dijimos, el supuesto que Toulmin y muchos otros filósofos de la ciencia del siglo Xx utilizan sistemáticamente como un principio metodológico básico es la oposición entre representaciones objetivas y representaciones subjetivas, no entre representaciones homológicas y otro tipo de representaciones.

A partir del giro historicista en los años sesenta, se acepta ampliamente la idea de que las representaciones científicas tienen que entenderse como "situadas" en un contexto histórico. En los estudios sobre la ciencia hay una gran variedad de sugerencias respecto a cómo entender las representaciones que coinciden en reconocer ese carácter situado y combinatorio del que hablan Ibarra y Mormann, pero que no parecen apoyar su clasificación $\mathrm{y}$, por lo tanto la manera de caracterizar formalmente las representaciones en la ciencia que ellos proponen. Ellos sugieren que las propuestas de Gallison, Latour, Pickering y muchos otros que han estudiado el tema de la representación en la ciencia apuntan al tipo de caracterización que ellos promueven. Creo, sin embargo, que esto es incorrecto, y que más bien esta gran variedad de estudios sobre la ciencia cuestionan seriamente el alcance del tipo de proyecto propuesto. Veamos.

La tesis de que hay una teoría filosófica general de las representaciones que "capta las propiedades esenciales de las representaciones" (p. $4, \S 2$ ) mejor que otras propuestas parte del supuesto, ciertamente controversial, de que existen tales propiedades esenciales de las representaciones. Toda una serie de investigaciones en estudios sobre la ciencia sugiere que no hay tal teoría 
general de la representación, y que la única manera fructífera de estudiar el tema de la representación es a través de un estudio de casos y de propuestas de clasificación de diferentes tipos de representación que puedan servir para entender mejor cómo crece el conocimiento. Esto no le quita interés filosófico al tema de la representación, pero lo sitúa en un espacio de discusión que no es el que los autores proponen. Sigue siendo filosóficamente interesante entender la manera en que determinados tipos de representación están asociados con diferentes tipos de conocimiento, por ejemplo. En todo caso, mi interés aquí no es defender una propuesta de ese tipo, sino más bien plantear mis dudas sobre un proyecto como el propuesto.

Hertz nos dice que hacemos imágenes internas de los objetos externos de manera tal que las consecuencias intelectualmente necesarias (denknotwendigen) de las representaciones (Bilder) son a su vez representaciones (Bilder) de las consecuencias naturalmente necesarias (naturnotwendingen). Los autores dicen que "aunque sugestiva en muchas ideas, la filosofía de la ciencia de Hertz está superada en aspectos esenciales", pero que tomar esta idea de la relación entre elementos intelectualmente necesarios y elementos naturalmente necesarios de Hertz no los compromete con esos aspectos ya superados (p. 13, §2). Los autores parecen creer que "naturalmente necesario" se elucida simplemente dándole el sentido de que el diagrama tipo Hertz (diagrama 2.1) conmuta. Pero, ¿por qué hacer esta identificación? Hertz tenía una base para hacer ese tipo de identificación: su firme creencia en el determinismo del mundo. No está claro que sin ese supuesto tenga mucho sentido el diagrama de Hertz.

Según los autores, la parte izquierda del diagrama $A \stackrel{t_{\mathrm{A}}}{\longrightarrow} A^{*}$ puede interpretarse como un proceso empírico en el que se pasa del estado $A$ al estado $A^{*}$ a través de $t_{\mathrm{A}}$, que a su vez se representa por un proceso teórico consistente en el paso de $B$ a $B^{*}$ por $t_{\mathrm{B}}$. Los autores consideran que $t_{\mathrm{B}}$ es una relación lógica entre $B$ y $B^{*}$ que corresponde a la relación $t_{\mathrm{A}}$ "naturalmente necesaria", en el sentido que el diagrama conmuta. Veamos qué puede querer decir esto en casos concretos. En el caso de un proceso caótico, las transformaciones de estado no son prede- 
cibles, y puede argüirse que esta falta de predictibilidad no es mero producto de nuestra ignorancia. En este caso, el paso de $A$ a $A^{*}$ es contingente, pero el paso de $B$ a $B^{*}$ no es contingente (se dice que es una relación lógica). Hay varias maneras de resolver este tipo de cuestiones, pero es importante decidirse por una manera de hacerlo para poder juzgar la propuesta. Un sistema caótico, por ejemplo, prima facie por lo menos, no parece adecuarse a representaciones descritas por el diagrama de Hertz. Algo tiene que decirse con respecto a la manera como se trata el tema de procesos no deterministas (e impredecibles). Por lo menos tan problemático sería el caso de una representación de un decaimiento radiactivo de acuerdo con la teoría cuántica. En este caso (según la mecánica cuántica), el paso del estado inicial al estado final no se describe mediante ninguna ecuación fundamental. ¿Cómo se entendería el diagrama de Hertz en este caso?

En relación con este punto, tengo otra preocupación. ¿Cuál es el concepto de estado en cuestión que en general estaría detrás de la identificación de un "estado" inicial $A$ del que se pasa a un estado $A^{*}$ ? El concepto de estado es un concepto bien definido en la mecánica newtoniana de partículas y en algunos otros tipos de teorías (que se prestan a una descripción de la dinámica en términos de espacios de fase), pero no en todas las teorías científicas tiene sentido hablar de estados, aunque metafóricamente pueda ser un concepto muy usado. Tampoco puede decirse que haya un concepto de estado que sea aplicable en toda la física, ni mucho menos en toda la ciencia. En la mecánica cuántica es bien sabido que uno de los problemas centrales de la interpretación de la teoría gira alrededor del concepto de estado. La tendencia predominante hoy en día es que es importante distinguir entre diferentes tipos de estado cuántico. Incluso en teorías clásicas de la física, el concepto ha sido puesto en entredicho. Hay propuestas de que el concepto de estado clásico debe desaparecer de la mecánica estadística (Prigogine). En resumen, el concepto de estado no puede darse por sentado en una discusión filosófica de teorías empíricas. O bien se propone una caracterización general del concepto de estado que pueda servir de base para una aplicación del diagrama 
de Hertz, que difícilmente tendría base en teorías científicas, o bien, se tiene que especificar caso por caso qué se entiende por el concepto de estado en cuestión, y en algunos casos tendrá sentido el diagrama de Hertz, y en otros no. Por supuesto que el hecho de que haya teorías que permitan sustentar la conmutatividad de las representaciones de acuerdo con el diagrama de Hertz no es para nada un resultado trivial, es un resultado importante que caracterizaría un tipo de representaciones que potencialmente es muy iluminador; pero esta pretensión sería diferente de la que los autores quieren sustentar.

Como ya lo mencioné, me parece que es indispensable entender más a fondo las diferencias entre tipos de representación, y, en particular, entender de manera concreta, a través de estudios detallados, las maneras en las que una representación es vicariante. Me parece difícil que haya tipos de representación vicariante que sean distintivos de la ciencia, y es por esto que el estudio del tema de la vicarianza es bastante complejo y tiene que involucrar tanto a las ciencias cognitivas como a la sociología (y a muchas otras disciplinas). En todo caso, creo que los estudios sobre la ciencia dejan claro que es necesario tomar mucho más en cuenta el carácter (o dimensión) vicariante (o sustitutivo, como lo llaman los autores) de una gran parte de las representaciones científicas. $^{2}$ En la medida en que no queda claro en la propuesta de los autores la manera en que las representaciones homológicas capturan la dimensión vicariante de (por lo menos muchas) representaciones científicas, la propuesta no llega a asentarse sobre terreno firme. ${ }^{3}$

2 Una dimensión que claramente no respeta la distinción tradicional entre representaciones "subjetivas" y "objetivas" (o por lo menos sugiere sentidos en los que es problemática). Es importante además reconocer que las representaciones "subjetivas" son importantes en la ciencia. Recientemente, Paul Thagard (1999, p. 163) arguye de manera convincente que hay importantes tipos de representaciones "subjetivas" que desempeñan un papel en la ciencia: "Although representational accounts of conceptual change do not tell the whole story, there remains ample reason to describe conceptual change in part as change in mental representations, specially kind relations."

3 Aunque creo que no es necesario para que se entienda mi crítica, quiero decir muy brevemente algo más sobre la manera de caracterizar las representaciones como poseedoras de dimensiones, en lugar de pensar en términos de 
Se dice en el texto de Ibarra y Mormann que la concepción predominante en la filosofía de la ciencia es la homomórfica (p. 19); sin embargo, esto no es del todo correcto. En todo caso, sería correcto si pensamos en la filosofía de la ciencia de corte formalista, en la tradición estructuralista, por ejemplo; pero si pensamos en la vertiente historicista de la filosofía de la ciencia es evidente que la dimensión vicariante y, por supuesto, la dimensión histórica de las representaciones está siempre presente y se resalta ampliamente de diferentes maneras. Tanto en Kuhn como en Feyerabend, Toulmin o Hacking, la dimensión vicariante de las representaciones es central para la caracterización que estos autores hacen de lo que es una representación científica.

$\mathrm{Y}$ si tomamos en cuenta propuestas en los estudios sobre la ciencia, como las de Galison, Pickering y Latour, veremos que la relación entre las diferentes dimensiones desempeña un papel central en el análisis. He aquí lo que dice Tibbets:

Clearly the concept of an isobar $[\ldots]$, rather than literally representing something, was simply borrowed from the fiction of contour lines on geological maps. What then do isobars (or contours lines) map onto? Nothing. On a constructivist account they are simply useful conceptual devices for introducing order into

formas de representación. Las representaciones suelen tener una dimensión vicariante, una dimensión homomórfica (posiblemente Ibarra y Mormann tienen razón cuando dicen que es mejor caracterizar esa dimensión como homológica), que de alguna manera cuantitativa o cualitativamente precisa captura semejanzas, y una dimensión histórica. Las tres dimensiones intervienen en el valor de uso y en el valor de cambio que tiene la representación. La dimensión vicariante tiene que ver con el sentido en el que una representación es una abstracción, abstracción que, en la terminología de Latour, la hace más "móvil". Las otras dos dimensiones contribuyen a hacer cuantitativa y cualitativamente precisa (para cierto público, i.e., en un determinado contexto públicamente determinable) la abstracción que representa (en el sentido de estar en el lugar de, en el sentido de "Darstellung"). El valor de uso tiene que ver con la capacidad de una representación de extender el dominio en el que cierta estructura explicativa, o un mecanismo, puede ayudarnos a entender cierta situación, que en la ciencia muchas veces consiste en modelar causalmente, o más en general, resolver o plantear un problema, o por lo menos ponerlo en perspectiva. El valor de cambio tiene que ver con la capacidad de la representación de ser comparada con otras representaciones, y a través de esa comparación someterse a una selección guiada por diferentes tipos de valores, epistémicos, morales, etc. Desarrollo esta idea en Filosofía de las prácticas científicas (inédito). 
measurements and, in turn, for extrapolating from past to future data points (Tibbetts 1988, pp. 73-74).

Ibarra y Mormann sugieren varias veces que enfoques como los de Pickering, Galison y Gooding deben verse como "primeros pasos" en la dirección de la concepción homológica. Según ellos, el trabajo de Pickering y Galison se "traduce" en la concepción homológica en la medida en que sus enfoques pueden entenderse como la búsqueda de hacer conmutativo el diagrama de Hertz. Explicar esto en detalle sería interesante, pero la mera afirmación de que así se puede entender a estos autores es poco útil, y para nosotros, los escépticos, es un problema serio. Ciertamente es correcto decir que "los enfoques que acaban de presentarse (Pickering y Galison) coinciden en reconocer la necesidad de un concepto más complejo y plural de representación para describir la compleja y plural práctica de las representaciones científicas" (p. 37). Esto es correcto, pero de aquí a que dichos enfoques apoyen el de Ibarra y Mormann hay un buen trecho. Existen muchas maneras de reconocer la complejidad de las prácticas representacionales de la ciencia, y en particular su carácter situado y combinatorio o seriado que no lleva al enfoque homológico. Es más, creo que si se ahonda un poco en lo que esos autores dicen, y en particular se reconoce la importancia que los estudios sobre la ciencia atribuyen a la dimensión (o, más apropiadamente, dimensiones) vicariante(s) de las representaciones, quedará bastante claro que estos enfoques no apoyan para nada el tipo de propuesta de Ibarra y Mormann. ${ }^{4}$

Como Toulmin ya lo recalca en Human Understanding, las representaciones en el sentido de "Darstellung" son representaciones vicariantes. Ni en Toulmin, ni en estudios contemporáneos sobre la ciencia (tales como los de Pickering, Latour o Galison), el hecho de que se reconozca la importancia de la dimensión vicariante de una representación implica negar su dimensión

4. Creo que reconocer la preeminencia o por lo menos la ubicuidad de la dimensión vicariante no iría en detrimento de la propuesta de Ibarra y Mormann, si se reformula de manera más modesta; pero sí iría directamente en contra de ella si se pretende que las representaciones sean reducibles a su dimensión homológica. 
homomórfica u homológica (véase la cita de Tibbetts supra). Galison por ejemplo deja muy claro que la tradición que busca representar imágenes de las cosas, lo que sería una versión de la tradición especular o isomórfica, tiene una dimensión vicariante y también una dimensión homomórfica (Galison 1997, p. 67).

Muchas representaciones en la ciencia son representaciones vicariantes en el sentido de representación objetiva del que hablan Hertz, Toulmin y Wittgenstein, pero esta vicarianza está ligada de manera importante a aspectos de una situación que se preservan a lo largo de una serie de transformaciones, aspectos que de alguna manera capturan semejanzas. Es importante recordar que reconocer la importancia y la ubicuidad de la dimensión vicariante de las representaciones científicas de ningún modo puede traducirse como una crítica a la idea de que las representaciones deben estudiarse en el contexto de otras representaciones. Muy por el contrario, nos permite entender mejor cómo es que series de representaciones tienen un papel tan importante en el desarrollo científico. Esta idea es, por ejemplo, el tema central de muchas de las contribuciones que aparecen en Michael Lynch y Steve Woolgar (comps.) 1988.

Para ver las implicaciones que para el proyecto de Ibarra y Mormann tiene este tipo de enfoques del tema de la representación, examinemos con un poco de detalle lo que dicen Latour y Galison, y la manera como quieren entenderlos Ibarra y Mormann.

Según Ibarra y Mormann, la propuesta de Latour de una "concepción deambulatoria de la referencia" (Latour 1999) fija una cadena de representaciones cuyos elementos, como en (3.4), ${ }^{5}$ pueden interpretarse como "pequeñas" representaciones. Si bien reconocen la importancia que Latour le otorga al carácter seriado de las representaciones, consideran que, a diferencia de la propuesta de Latour, que según ellos sólo se limita al tipo de combinaciones lineales ejemplificada en (3.4), su propuesta admite combinaciones no-lineales. Los autores no dicen mucho más respecto a las similitudes y las diferencias de su propuesta

${ }^{5}$ Véase Crítica, no. 95, p. 22. 
con la de Latour, pero es instructivo ahondar en esta comparación, sobre todo porque, a diferencia de ellos, Latour desarrolla sus ideas usando ejemplos bastante elaborados y como veremos, tiene una idea muy diferente de lo que es una representación en la ciencia.

Las representaciones de Latour son fotografías, diagramas, gráficas, resultados de mediciones de instrumentos y muchas otras cosas que a través de su comparación y decantamiento (abstracción) van construyendo representaciones "móviles", transportables, que permiten la comparación y el contraste con otras representaciones. No hay nada privilegiado, dice Latour, en el paso a las palabras, todo tipo de representación puede servir para capturar la referencia, lo importante es la manera en la que se alinean las diferentes representaciones de modo tal que principiando con la última representación seamos capaces de regresar a la primera (Latour 1999, p. 64).

En Latour, las representaciones se alinean, y es este alineamiento lo que permite que sean generadoras de conocimiento. Dice Latour que esta relación de representación o delegación tan bien regulada no puede entenderse para nada como mímesis, o correspondencia, pero también dice que reconocer esa concatenación de representaciones como conocimiento requiere movimientos mucho más confiables que la mera correspondencia, contrastes y comparaciones que tienen lugar a través de capas de transformaciones, movimientos como los de un cangrejo, indirectos y cruzados (Latour 1999, p. 64). 6 Para Latour es muy importante construir la dirección de una cadena de representaciones a fin de entender cómo se construyen los "inmutables móviles", y por lo tanto el conocimiento. Pero el reconocimiento de esta direccionalidad para nada excluye que las series de representaciones diverjan en algún punto. Es más, Latour reconoce que esto sucede muchas veces, que, por ejemplo, cierta representación puede ser el punto de partida de muchas series de representaciones. El alineamiento de las tradiciones no implica

6 Por cierto, nótese que esta manera de hablar tiene sentido en términos del modelo dimensional de las representaciones, pero no si se entienden las representaciones como homologías. 
el carácter "lineal" de las series de representaciones. Es evidente entonces que es incorrecto atribuirle a Latour una restricción con cadenas lineales de representaciones; en todo caso, la diferencia de los autores con Latour está en otra parte.

En la concepción de representación de Latour, la dimensión vicariante desempeña un papel central. Latour dice con respecto de una representación: "it does not resemble anything. It does more than resemble. It takes the place of the original situation, which we can retrace, [...]" (p. 67). Una propiedad crucial de esta cadena de representaciones es que debe ser reversible. Toda serie fluye hacia arriba y hacia abajo. Si la cadena se interrumpe en cualquier punto, deja de transportar la referencia. Los fenómenos son precisamente, para Latour, lo que circula en esas cadenas reversibles de transformaciones. ¿Es esta reversibilidad la que se pretende capturar con el diagrama de Hertz? De ser así sería interesante ver cómo se pretende hacerlo; vagas sugerencias al respecto no ayudan.

Sin embargo, por lo menos prima facie esta manera de reformular la idea de Latour no es plausible. La referencia - dice Latour- no es simplemente el acto de apuntar o una manera de recolectar garantías de la verdad de un enunciado, sino una manera de mantener algo constante a través de una serie de transformaciones. Y continúa: "Knowledge does not reflect a real external world that it resembles via mimesis, but rather a real interior world, the coherence and continuity of which it helps to ensure" (Latour 1999, p. 58). Esta idea recurre de manera contundente a la dimensión vicariante o sustitutiva de la representación, así como a la dimensión homomórfica, e implícitamente rechaza la pretensión de que las representaciones son "objetivas" en oposición a "subjetivas". Esto no concuerda con una concepción de la representación como homología que parece tener que comprometerse con la distinción tradicional entre representaciones objetivas y subjetivas, o que por lo menos parece suponer que las representaciones de interés en la filosofía de la ciencia son sólo las "objetivas".

Como ya lo vimos en el caso de Toulmin, la tesis de la interdependencia serial de las representaciones es independiendente de la tesis de que las representaciones deben entenderse como 
homologías, incluso reconociendo que las representaciones deben entenderse en el sentido de Hertz, en el sentido de "Darstellung"; e incluso si se admite la distinción tradicional entre representaciones objetivas y subjetivas, y se acepta que la filosofía de la ciencia sólo trata de representaciones objetivas en el sentido de públicas.

Veamos ahora el caso de Galison. Según Ibarra y Mormann, Galison, como otros autores de estudios sobre la ciencia, remarca "la dificultad de la construcción de un diagrama de Hertz, esto es, la dificultad de constituir un equilibrio entre práctica, instrumentación, interpretación y teoría" (p. 33). Es más, atribuyen a Galison y a otros autores la tesis de que la construcción de un diagrama de Hertz es el fin explícito de toda actividad científica. Por supuesto que Galison y Latour nunca dicen nada parecido; en todo caso, lo que dicen se podría interpretar de esa manera, pero eso está por verse. Como hemos visto en el caso de Latour, y como veremos ahora en el caso de Galison, hay buenas razones para pensar que esta manera de reformular esos enfoques no es correcta.

En primer lugar es importante recordar que Galison no pretende que la tradición mimética y la tradición lógica en la física del siglo XX sean tradiciones o formas de representar subespecie aeternitatis. Además, algo que Galison deja claro es que para él la conexión entre lo específico y lo general no es metonímica: "la muestra (el caso) no es una muestra representativa del todo". Lo que caracteriza historias como la que presenta en Image and Logic (o como las de Carlo Ginzburg que utiliza como ejemplo) es el uso de lo local y lo específico para explorar más allá de lo próximo, a través de un tanteo en el uso de símbolos, significados y valores tal y como éstos se expresan en el ámbito restringido de culturas particulares (1997, p. 61). En este mismo sentido Galison presenta su distinción entre dos tradiciones de representación en el siglo XX:

Our map is not one of conquering armies or spreading disease launched from one side and dominating all it encounters. Instead the limit zones between the various industrial, military, experimental, instrumental, and theoretical pieces of physics have complex and 
local boundary conditions: not the mathematically thin boundaries of topology, but the substantive productive boundaries that Peter Sahlins so effectively describes in the Pyrenees. There are enclaves, and intermediate communities, jointly administered sectors, borderland languages, and autonomy movements at the margin (Galison 1997, p. 53).

Esta manera historicista de caracterizar las diferentes tradiciones de representación no concuerda con el tipo de enfoque que Ibarra y Mormann persiguen, y en realidad es totalmente ajena a él, es más, creo que por las mismas razones que Galison rechaza que lo que escribe sea una "historia de caso" más, debe rechazarse la idea de que estas dos tradiciones que Galison identifica logren encajar en la clasificación de Ibarra y Mormann (y en particular modelarse como representaciones homológicas). Galison dice:

But I resist the designation "case study" because I do not believe that there is a set of defining precepts that can be abstracted from these or other studies to "experiment in general" (or for that matter, "theory in general", or "instruments in general"). Experimentation is so rich and varied [...] that I have no interest in pronouncements about putatively time-trascendent principles of laboratory action" (1997, p. 62).

Queda claro, entonces, que la pretensión de los autores de interpretar a Galison como si elaborara "la significación universal de las representaciones materiales" (p. 36) está profundamente desencaminada.

En conclusión, he argüido que la tesis de que las representaciones en la ciencia "presentan el formato característico de las representaciones homólogas" está lejos de poder apoyarse en los importantes estudios sobre la naturaleza de la representación científica que ellos mismos citan. He hecho ver también que la clasificación que Ibarra y Mormann utilizan como punto de partida no parece corresponder a la manera en que de hecho intervienen las representaciones en la práctica científica. 


\section{BIBLIOGRAFÍA}

Galison, P., 1997, Image and Logic. A Material Culture of Microphysics, The University of Chicago Press, Chicago.

Goodman, N., 1972, Problems and Projects, Bobbs-Merrill, Indianápolis.

Hertz, H., 1894, Die Prinzipien der Mechanik in neuem Zusammenhange dargestellt, 2a. ed., preparada por P. Lenard, Leipzig.

Ibarra, A. y T. Mormann, "Una teoría combinatoria de las representaciones científicas", Crítica, vol. XXXII, no. 95, 2000, pp. 3-46.

Latour B., 1999, Pandora's Hope. Essays on the Reality of Science Studies, Harvard University Press, Cambridge, Mass.

Lynch M. y S. Woolgar, 1988, Representation in Scientific Practice, Human Studies, vol. 11, no. 2-3 (abril-julio).

Thagard, P., 1999, How Scientists Explain Disease, Princenton University Press, Princeton, Nueva Jersey.

Tibbetts, P., 1988, "Representation and the Realist-Constructivist Controversy", en M. Lynch y S. Woolgar 1988.

Toulmin, S., 1972, Human Understanding, Princeton University Press, Princeton, Nueva Jersey.

Recibido el 6 de marzo de 2001; aceptado el 23 de agosto de 2001 